\title{
Anisotropic spin and charge excitations in superconductors: Signature of electronic nematic order
}

\author{
Ying-Jer Kao ${ }^{1,2}$ and Hae-Young Kee ${ }^{1}$ \\ ${ }^{1}$ Department of Physics, University of Toronto, Toronto, Ontario M5S 1A7, Canada \\ ${ }^{2}$ Department of Physics, National Taiwan University, Taipei 106, Taiwan
}

(Received 14 December 2004; published 1 July 2005)

\begin{abstract}
We study spin and charge susceptibilities in the $d$-wave superconducting state whose underlying electronic dispersion is anisotropic due to the formation of electronic nematic order. We show that the amplitude of the incommensurate peaks in the spin susceptibility near $(\pi, \pi)$ reveals a pronounced anisotropy in momentum space. The relevance of our findings to the magnetic scattering pattern observed in recent neutron scattering measurements on untwinned $\mathrm{YBa}_{2} \mathrm{Cu}_{3} \mathrm{O}_{6+x}$ is discussed. In the charge channel, we identify a well-defined collective mode at small momentum transfer with strong anisotropic amplitude depending on the direction of the momentum transfer, which is associated with the broken symmetry due to nematic ordering.
\end{abstract}

DOI: 10.1103/PhysRevB.72.024502

PACS number(s): 74.20.-z, 71.10.Hf, 71.27.+a, 71.45.Gm

\section{INTRODUCTION}

It has been recently proposed that the quantum analog of liquid crystal states-dubbed electronic smectic and nematic phases-may play an important role in the physics of strongly correlated systems. ${ }^{1-7}$ The smectic and nematic phases are characterized by the broken unidirectional translational and rotational symmetry and broken rotational symmetry, respectively. When electrons reside on a lattice, the broken translational and rotational symmetries correspond to the broken point-group symmetry of the underlying lattice. For example, the electronic nematic phase is characterized by the broken $x-y$ symmetry on a square lattice and the collective modes associated with the broken symmetry. Direct or indirect evidence of electronic smectic and nematic phases has been reported in various strongly correlated systems, via different experimental techniques. ${ }^{3-9}$

In particular, a recent neutron scattering measurement on untwinned $\mathrm{YBa}_{2} \mathrm{Cu}_{3} \mathrm{O}_{6+x}$ has indicated the possibility of a nematic liquid phase in high-temperature superconductors. ${ }^{10}$ Neutron scattering experiments, which provide direct information on the spin dynamics as well as static magnetic order, have been widely and successfully used to understand novel phenomena in high-temperature superconductors. However, theoretical interpretations of the origin of incommensurate spin excitations observed by neutron scattering experiments are still controversial. ${ }^{11}$ One explanation of this phenomenon is that it is due to Fermi surface nesting. ${ }^{12}$ However, it was also proposed that this phenomenon can be understood due to the formation of one-dimensional stripes in the system. ${ }^{1,8}$ A recent neutron experiment ${ }^{10}$ shows that the spin fluctuations in this material are indeed of two-dimensional nature: the locus of maximum spin fluctuation spectral weight approximately forms a circle in momentum space. However, there exists a strong anisotropy in the amplitude and width of the incommensurate peaks depending on the direction of the momentum transfer. These experimental results may indicate that the electronic structure in the high-temperature superconductors is neither rigid one-dimensional stripe arrays nor an isotropic two-dimensional system.
In this paper, we study incommensurate spin and charge fluctuations in $d$-wave superconducting states which coexist with the electronic nematic phase. We find that the strong intensity anisotropy in the incommensurate spin susceptibility near $(\pi, \pi)$ is due to the anisotropic electronic dispersion of the nematic phase. The incommensurabilities and width of the incommensurate peaks also show anisotropy in momentum space. The qualitative results bear a resemblance to the magnetic scattering pattern observed in neutron scattering measurements. ${ }^{10,13}$ We also study the charge dynamics, especially focus on the effects of coupling to the collective modes. We find that a pronounced peak appears at moderate energies for small momentum transfer. This peak is an overdamped collective mode of the amplitude fluctuations at extremely small wave vectors and grows into a sharp mode at moderate finite wave vectors. The intensity of this mode also shows anisotropy in the momentum plane, which results from the broken $x-y$ symmetry of the nematic order.

This paper is organized as follows. In the following section, we describe the nature of nematic order and introduce an effective Hamiltonian for the nematic transition within the weak-coupling theory. We also give a brief summary for completeness of the mean-field results of the effective Hamiltonian presented previously. ${ }^{14,15}$ We present spin and charge susceptibilities in the superconducting state which occur on top of the nematic phase in Secs. III and IV, respectively. The connection to experimental observations is discussed in Sec. V. The conclusion and summary are given in Sec. VI.

\section{MODEL}

For classical liquid crystals, the nematic order parameter is represented by a director, which is a quadrupolar (rank-2 symmetric traceless) tensor built from the spatial directions. In two dimensions, it changes sign under a rotation of $\pi / 2$ and is invariant under a rotation of $\pi$. In a similar spirit, one can construct a quadrupolar order parameter for electronic systems using the momentum operators of electrons, $\hat{Q}_{i j}$ 
$=\hat{p}_{i} \hat{p}_{j}-\frac{1}{2} \hat{p}^{2} \delta_{i j}$. The attractive interaction between quadrupole densities can lead to the nematic order which breaks the rotational symmetry, and a preferred direction for the electron momenta occurs.

Within the weak-coupling theory, the following effective Hamiltonian of the quadrupole-quadrupole density interaction has been proposed to describe the transition between isotropic liquid to the nematic-ordered phase, ${ }^{14-17}$ where the expectation value of the quadrupole density serves as the order parameter of the nematic phase,

$$
\mathcal{H}=\sum_{\mathbf{k}} \sum_{\sigma=\uparrow, \downarrow} \epsilon_{\mathbf{k}} c_{\mathbf{k}, \sigma}^{\dagger} c_{\mathbf{k}, \sigma}+\sum_{\mathbf{q}} F_{2}(\mathbf{q})\left\{\operatorname{Tr}\left[\hat{Q}_{\sigma}^{\dagger}(\mathbf{q}) \hat{Q}_{\sigma}(\mathbf{q})\right]\right\},
$$

where $\epsilon_{\mathbf{k}}=-2 t\left(\cos k_{x}+\cos k_{y}\right)-4 t^{\prime} \cos k_{x} \cos k_{y}-\mu$ is the bare electronic dispersion. $F_{2}(\mathbf{q})$ is a short-range attractive interaction, which can be written in a general form as

$$
F_{2}(\mathbf{q})=-\frac{F_{2}}{1+\kappa F_{2} q^{2}},
$$

where $F_{2}>0 . \hat{Q}(\mathbf{q})$ is the quadrupole density tensor,

$$
\hat{Q}_{\sigma}(\mathbf{q})=\sum_{\mathbf{k}} c_{\mathbf{k}+\mathbf{q}, \sigma}^{\dagger}\left(\begin{array}{ll}
F(k, q) & G(k, q) \\
G(k, q) & F(k, q)
\end{array}\right) c_{\mathbf{k}, \sigma},
$$

where $\quad F(k, q)=\left\{\left(\cos k_{x}-\cos k_{y}\right)+\left[\cos \left(k_{x}+q_{x}\right)-\cos \left(k_{y}\right.\right.\right.$ $\left.\left.\left.+q_{y}\right)\right]\right\} / 2$ and $G(k, q)=\left\{\left(\sin k_{x} \sin k_{y}\right)+\left[\sin \left(k_{x}+q_{x}\right) \sin \left(k_{y}\right.\right.\right.$ $\left.\left.\left.+q_{y}\right)\right]\right\} / 2$. Notice that $\hat{Q}^{\dagger}(\mathbf{q})=\hat{Q}(-\mathbf{q})$, indicating that $\hat{Q}(r)$ is Hermitian.

The mean-field solution has already been presented in Refs. 14 and 15, and we give a brief summary of the result here for completeness. To solve the model at the mean-field level, we decouple the interaction at $\mathbf{q} \rightarrow 0$ and define two order parameters

$$
\begin{aligned}
& \Delta_{N}=F_{2}\left\langle Q_{x x}(0)\right\rangle=F_{2} \sum_{k} f\left(\tilde{\boldsymbol{\epsilon}}_{k}\right) F(k), \\
& \Delta_{N}^{\prime}=F_{2}\left\langle Q_{x y}(0)\right\rangle=F_{2} \sum_{k} f\left(\widetilde{\boldsymbol{\epsilon}}_{k}\right) G(k),
\end{aligned}
$$

where $F(k)=\left(\cos k_{x}-\cos k_{y}\right)$ and $G(k)=\sin k_{x} \sin k_{y}$. The electron dispersion in the nematic phase is renormalized to

$$
\begin{aligned}
\tilde{\epsilon}_{\mathbf{k}}= & -2 t\left(1-\Delta_{N} / 2 t\right) \cos k_{x}-2 t\left(1+\Delta_{N} / 2 t\right) \cos k_{y} \\
& -4 t^{\prime} \cos k_{x} \cos k_{y}-\Delta_{N}^{\prime} \sin k_{x} \sin k_{y}-\mu .
\end{aligned}
$$

Nonzero values of $\Delta_{N}$ and $\Delta_{N}^{\prime}$ indicate the lattice rotational symmetry is broken and there exists a preferred direction for the electron momentum. At the mean-field level, with a single value of $F_{2}$, it is found that $\Delta_{N}^{\prime}$ always vanishes, which indicates that the deformation of the Fermi surface is along the underlying lattice axes. It is also found that $\Delta_{N}$ jumps at the transition from the isotropic phase to the nematic phase by tuning the chemical potential and/or the strength of the interaction, which leads to a dramatic change of the Fermi surface topology - a closed to an open Fermi surface-at the transition. Therefore, the nematic-ordered state describes electrons with anisotropic hopping and the topology of the
Fermi surface changes dramatically at the transition. ${ }^{14,15}$

Fluctuations around the mean-field states can be described by amplitude and phase (i.e., orientational) deformations of the order parameter, and we obtain the collective mode spectrum at the Gaussian level. In the continuum case, the phase mode is an overdamped Goldstone mode and the amplitude mode is gapped. ${ }^{17}$ In the lattice case, the nematic order is pinned to the underlying lattice, so the phase mode is also gapped. The form factors associated with the amplitude and phase fluctuations are $F(k, q)$ and $G(k, q)$, respectively. ${ }^{18}$

In the following sections, we study the spin and charge susceptibilities in the $d$-wave superconducting state, which occurs on top of the nematic liquid with anisotropic dispersion. The quasiparticle dispersion in the superconducting state is given by $E_{k}=\sqrt{\widetilde{\epsilon}_{k}^{2}+\Delta_{k}^{2}}$, with $\widetilde{\epsilon}_{k}$ the electronic dispersion in the nematic phase given by Eq. (4) and $\Delta_{k}$ $=\Delta_{s c}\left(\cos k_{x}-\cos k_{y}\right) / 2$ the $d$-wave superconducting gap.

\section{DYNAMICAL SPIN SUSCEPTIBILITY}

To analyze the dynamical spin susceptibility, we employ the standard random phase approximation (RPA) at the exchange interaction and the quadrupole density interaction. The spin susceptibility in the RPA is given by

$$
\chi^{s}(q, \omega)=\frac{\chi_{0}^{s}}{1+J(q) \chi_{0}^{s}},
$$

where $J(q)=J\left(\cos q_{x}+\cos q_{y}\right) / 2$ is the exchange interaction and $\chi_{0}^{s}$ is the susceptibility irreducible to $J(q) \cdot \chi_{0}^{s}$ includes the bare spin susceptibility as well as the coupling between the spin density and the collective modes of the nematic order, which can be written as ${ }^{19}$

$$
\chi_{0}^{s}=\chi_{00}^{s}-\sum_{a=1,2} \frac{V_{a}^{s} F_{2}(q) \bar{V}_{a}^{s}}{1+F_{2}(q) \Pi_{a}^{s}} \equiv \chi_{00}^{s}+\chi_{N}^{s},
$$

where $\chi_{00}^{s} \sim\left\langle\left[S_{z}, S_{z}\right]\right\rangle_{0}$ is the bare spin susceptibility, $V_{a}^{s}$ $\sim\left\langle\left[S_{z}, \delta \Delta_{N, z}^{a}\right]\right\rangle_{0}, \bar{V}_{a}^{s} \sim\left\langle\left[\delta \Delta_{N, z}^{a}, S_{z}\right]\right\rangle_{0}$ represents the coupling between the spin density and nematic order fluctuations, and $\Pi_{a}^{s} \sim\left\langle\left[\delta \Delta_{N, z}^{a}, \delta \Delta_{N, z}^{a}\right]\right\rangle_{0}$ is the correlation between nematic order fluctuations. Here $a=1,2$ corresponds to the amplitude and phase mode, respectively, and $\Delta_{N, z}=\Delta_{N, \uparrow}-\Delta_{N, \downarrow}$. In the superconducting state, these quantities are given by the BCStype expression assuming that the $d$-wave superconductivity is built on top of the nematic phase,

$$
\begin{aligned}
\chi_{00}^{s}, & V_{a}^{s}, \Pi_{a}^{s}(q, \omega) \\
& =\sum_{k} M(k, q)\left[\frac{1}{2}(1\right. \\
& +\frac{\left.\left.\widetilde{\epsilon}_{k} \widetilde{\epsilon}_{k+q}+\Delta_{k} \Delta_{k+q}\right) \frac{f\left(E_{k+q}\right)-f\left(E_{k}\right)}{E_{k} E_{k+q}}\right)\left(E_{k+q}-E_{k}\right)+i \delta}{\omega-f\left(E_{k+q}\right)-f\left(E_{k}\right)} \\
& +\frac{1}{4}\left(1-\frac{\tilde{\epsilon}_{k} \widetilde{\epsilon}_{k+q}+\Delta_{k} \Delta_{k+q}}{E_{k} E_{k+q}}\right) \frac{1-\left(E_{k+q}+E_{k}\right)+i \delta}{\omega+f\left(E_{k}\right)-1} \\
& \left.+\frac{1}{4}\left(1-\frac{\tilde{\epsilon}_{k} \tilde{\epsilon}_{k+q}+\Delta_{k} \Delta_{k+q}}{E_{k} E_{k+q}}\right) \frac{f\left(E_{k+q}\right)+f}{\omega-\left(E_{k+q}+E_{k}\right)+i \delta}\right],
\end{aligned}
$$




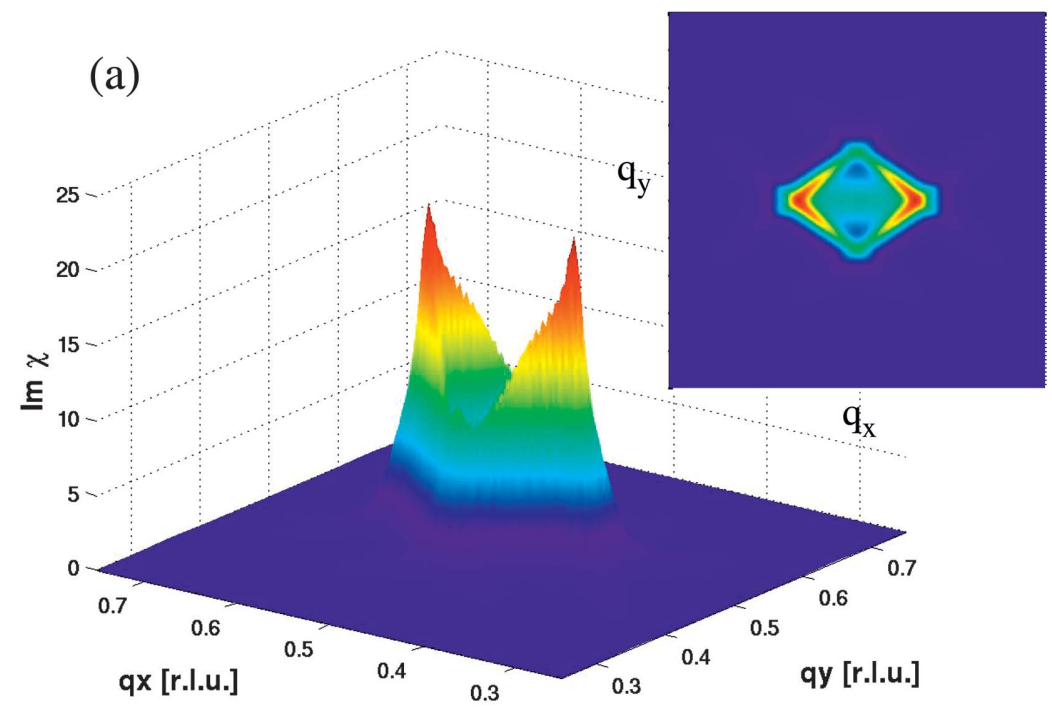

FIG. 1. (Color) Imaginary part of the spin susceptibility at $\omega / 2 t$ $=0.12$. (a) Three-dimensional (3D) plot of $\operatorname{Im} \chi$ shows strong amplitude anisotropy in the $q_{x}-q_{y}$ plane near $(\pi, \pi)$. (Inset) 2D plot of $\operatorname{Im} \chi$. The incommensurability pattern forms a "diamond" shape. (b) Line scans of $\operatorname{Im} \chi$ at different $q_{x}$ values. The incommensurabilities are $\delta_{x}=0.08$ and $\delta_{y}$ $=0.07$ r.l.u.

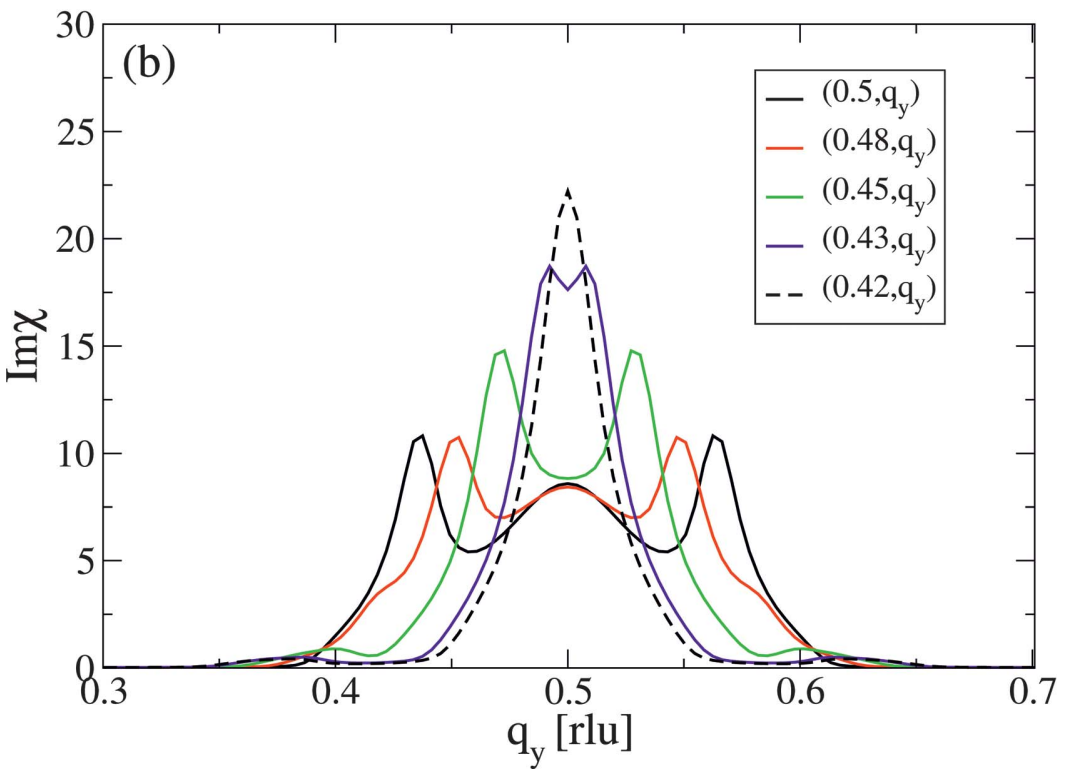

where $\tilde{\epsilon}_{k}$ is the anisotropic dispersion given by Eq. (4), $\Delta_{k}$ $=\Delta_{s c}\left(\cos k_{x}-\cos k_{y}\right) / 2$ is the $d$-wave superconducting gap, $E_{k}=\sqrt{\widetilde{\epsilon}_{k}^{2}+\Delta_{k}^{2}}$ is the quasiparticle energies, $f$ is the Fermi function, and $M(k, q)$ are form factors given by

$$
M(k, q)= \begin{cases}1 & \text { for } \chi_{00}^{s}, \\ F(k, q) & \text { for } V_{1}^{s}, \\ G(k, q) & \text { for } V_{2}^{s}, \\ F^{2}(k, q) & \text { for } \Pi_{1}^{s}, \\ G^{2}(k, q) & \text { for } \Pi_{2}^{s} .\end{cases}
$$

For the bare electron dispersion, we use the tight-binding parameters in Ref. 20, given by $t=138 \mathrm{meV}, t^{\prime}=-0.24 t$, and $\mu=-0.634 t$. The superconducting gap is $\Delta_{s c}=0.252 t$ $\approx 35 \mathrm{meV}$. The choice of $J=t$ gives the resonance at $(\pi, \pi)=(0.5,0.5)$ reciprocal-lattice units (r.l.u.) at $\Omega_{0}=0.29 t$ $\approx 40 \mathrm{meV}$, corresponding to the resonance peak observed in optimally doped $\mathrm{YBa}_{2} \mathrm{Cu}_{3} \mathrm{O}_{6+x}$. We take the nematic order parameter, $\Delta_{N} / 2 t=0.1$, temperature $T / 2 t=0.005$, and $\delta / 2 t$ $=0.005$ in the numerical calculations. The momentum integrals are summed over a $512 \times 512$ mesh in the Brillouin zone. We set the parameters in $F_{2}(\mathbf{q})$ to be $F_{2} / 2 t=0.49$ and $\kappa=1$. This set of $F_{2}, \mu$, and $\Delta_{N}$ satisfies the mean-field equation of Eq. (3).

Figure 1 shows the imaginary part of the RPA spin susceptibility at $\omega=0.24 t \approx 33 \mathrm{meV}$, slightly below the resonance. A few important observations follow: first, the incommensurate peaks in the nematic state show clear anisotropy along the $\left(\pi, \pi \pm \delta_{y}\right)$ and $\left(\pi \pm \delta_{x}, \pi\right)$ directions. In Fig. 1(b), we show several $q_{y}$ line scans at different fixed $q_{x}$ values. The amplitude of the incommensurate peaks is small initially along the $q_{y}$ direction and continues to grow until finally the amplitude becomes maximum along the $q_{x}$ direction. Second, the incommensurabilities are given by $\delta_{x}=0.08$ and $\delta_{y}$ $=0.07$ r.l.u. The incommensurability pattern shows a diamond shape, slightly elongated along the $q_{x}$ direction, which is the direction of the elongated Fermi surface due to nematic ordering [Fig. 1(a), inset]. Third, there is a strong anisotropy 


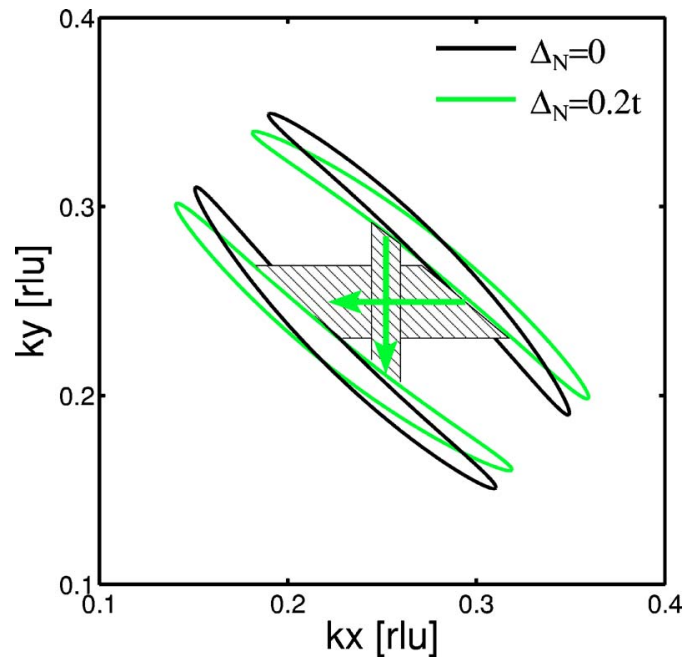

FIG. 2. (Color online) Energy contour plots for the quasiparticle dispersion $E_{k}$ and $E_{k+Q}=\omega / 2=0.12 t$ in the $d$-wave superconducting state. Black (dark) and green (light) curves are contours with $\Delta_{N} / 2 t=0$ and $\Delta_{N} / 2 t=0.1$, respectively. Green arrows are vectors with the same length. In the nematic phase, rotational symmetry is broken, so the nesting vector for the vertical shift is no longer a nesting vector for the horizontal shift. And the difference in the nesting areas (shaded areas) results in the amplitude anisotropy.

in the width of the incommensurate peaks. The ratio between the full width of half maximum of the incommensurate peak at $(0.42,0.5)$ and that at $(0.5,0.43)$ is about 0.58 . Finally, the tendency of anisotropy in the incommensurate peaks depends on the frequency. At higher frequencies, closer to the resonance mode, the anisotropy gets weaker and disappears at the resonance mode around $40 \mathrm{meV}$. These results bear a resemblance to the magnetic scattering pattern observed in recent neutron scattering experiments in $\mathrm{YBa}_{2} \mathrm{Cu}_{3} \mathrm{O}_{6+x^{*}}{ }^{10,13}$ However, the locus of the maximum spectral weight shows approximately circular shape in experimental data, ${ }^{10}$ while we find a diamond-like pattern, which is similar to that found in Ref. 20 based on the same set of parameters for the bare band structure.

We find that the major contribution of the anisotropy of amplitude and width of incommensurate peaks in momentum space comes from the bare spin susceptibility $\chi_{00}^{s}$ due to the anisotropic band structure, while the contribution from the coupling to the collective modes, $\chi_{N}^{s}$, is negligible, because around $\mathbf{q}=(\pi, \pi), V_{a}^{s}$ are small due to the form factors.

The anisotropy of the amplitude and incommensurability can be understood from the quasiparticle energy dispersion $E_{k}$. At low temperatures, we notice $\operatorname{Im} \chi_{00}^{s} \sim \Sigma_{k} \delta\left(\omega-E_{\mathbf{k}}\right.$ $+E_{\mathbf{k}+\mathbf{q}}$ ) apart from the coherence factors. The major contribution of the incommensurability is due to the nesting between the energy contour around the node, $E_{\mathbf{k}} \sim \omega / 2$ $\approx 0.12 t$, to the same contour displaced by $\mathbf{Q}=(\pi, \pi), E_{\mathbf{k}+\mathbf{Q}}$ $\sim \omega / 2 .^{21}$ The best nesting vector is horizontal or vertical offset to $(\pi, \pi)$, at $\mathbf{q}=\left(\pi \pm \delta_{x}, \pi\right),\left(\pi, \pi \pm \delta_{y}\right) \equiv(\pi, \pi)+\delta \mathbf{q} .{ }^{22}$ In Fig. 2, we plot the energy contours for $E_{\mathbf{k}}, E_{\mathbf{k}+\mathbf{Q}}=\omega / 2$ $=0.12 t$ for the isotropic case (black curves) and the nematic case (green curves). For the isotropic dispersion, horizontal and vertical incommensurabilities are equal. On the other hand, for the anisotropic dispersion due to the nematic ordering, the $x-y$ rotational symmetry is broken and a preferred direction is selected. In Fig. 2, the vertical arrow corresponds to a nesting vector $\delta \mathbf{q}=(0, \delta)$ and the horizontal arrow corresponds to the same vector rotated by $90^{\circ}[(\delta, 0)]$. However, with finite $\Delta_{N}$, the vector $(\delta, 0)$ is no longer a nesting vector, which explains why the locus of maximum spin spectral weight occurs at slightly different incommensurate vectors $\delta_{x}$ and $\delta_{y}$. The nesting regions for the horizontal and vertical offset vectors (Fig. 2, shaded areas) become different due to the nematic order, which results in the pronounced anisotropy in the amplitudes of the incommensurate peaks.

\section{DYNAMICAL CHARGE SUSCEPTIBILITY}

To analyze the dynamical charge susceptibility, we employ the RPA at the quadrupole density interaction and onsite Coulomb interaction. Similar to the previous section, the charge susceptibility in the RPA is given by

$$
\chi^{c}(q, \omega)=\frac{\chi_{0}^{c}}{1+U \chi_{0}^{c}},
$$

where $U=2 t$ is the on-site Coulomb interaction and $\chi_{0}^{s}$ is the susceptibility irreducible to $U$. $\chi_{0}^{c}$ includes the bare charge susceptibility $\chi_{00}^{c}$, as well as the coupling between the density and the nematic order, which can be written as follows:

$$
\chi_{0}^{c}=\chi_{00}^{c}-\sum_{a=1,2} \frac{V_{a}^{c} F_{2}(q) \bar{V}_{a}^{c}}{1+F_{2}(q) \Pi_{a}^{c}} \equiv \chi_{00}^{c}+\chi_{N}^{c},
$$

where $\chi_{00}^{c} \sim\langle[\rho, \rho]\rangle_{0}$ is the bare density susceptibility, $V_{a}^{c}$ $\sim\left\langle\left[\rho, \delta \Delta_{N}^{a}\right]\right\rangle_{0}$ and $\bar{V}_{a}^{c} \sim\left\langle\left[\delta \Delta_{N}^{a}, \rho\right]\right\rangle_{0}$ represent the coupling between the density and nematic order fluctuations, and $\Pi_{a}^{c}$ $\sim\left\langle\left[\delta N_{a}, \delta N_{a}\right]\right\rangle_{0}$ the correlation between nematic order fluctuations.

In the superconducting state, these quantities are given by the BCS-type expression, assuming that the superconductivity occurs on top of the nematic phase,

$$
\begin{aligned}
\chi_{00}^{c}, & V_{a}^{c}, \Pi_{a}^{c}(q, \omega)=\sum_{k} M(k, q)\left[\frac{1}{2}(1\right. \\
+ & \left.\frac{\tilde{\epsilon}_{k} \tilde{\epsilon}_{k+q}-\Delta_{k} \Delta_{k+q}}{E_{k} E_{k+q}}\right) \frac{f\left(E_{k+q}\right)-f\left(E_{k}\right)}{\omega-\left(E_{k+q}-E_{k}\right)+i \delta} \\
& +\frac{1}{4}\left(1-\frac{\widetilde{\epsilon}_{k} \widetilde{\epsilon}_{k+q}-\Delta_{k} \Delta_{k+q}}{E_{k} E_{k+q}}\right) \frac{1-f\left(E_{k+q}\right)-f\left(E_{k}\right)}{\omega+\left(E_{k+q}+E_{k}\right)+i \delta} \\
& \left.+\frac{1}{4}\left(1-\frac{\widetilde{\epsilon}_{k} \widetilde{\epsilon}_{k+q}-\Delta_{k} \Delta_{k+q}}{E_{k} E_{k+q}}\right) \frac{f\left(E_{k+q}\right)+f\left(E_{k}\right)-1}{\omega-\left(E_{k+q}+E_{k}\right)+i \delta}\right],
\end{aligned}
$$

and the form factors $M(k, q)$ have the same definition as in Eq. (8).

We show in Fig. 3 the imaginary part of the RPA charge susceptibilities $\chi^{c}$ in the $\left(q_{x}, 0\right)$ and $\left(0, q_{y}\right)$ directions at small momentum transfer $\mathbf{q}$. There is a peak at low energy and a broader spectrum at higher energies. We show the momen- 

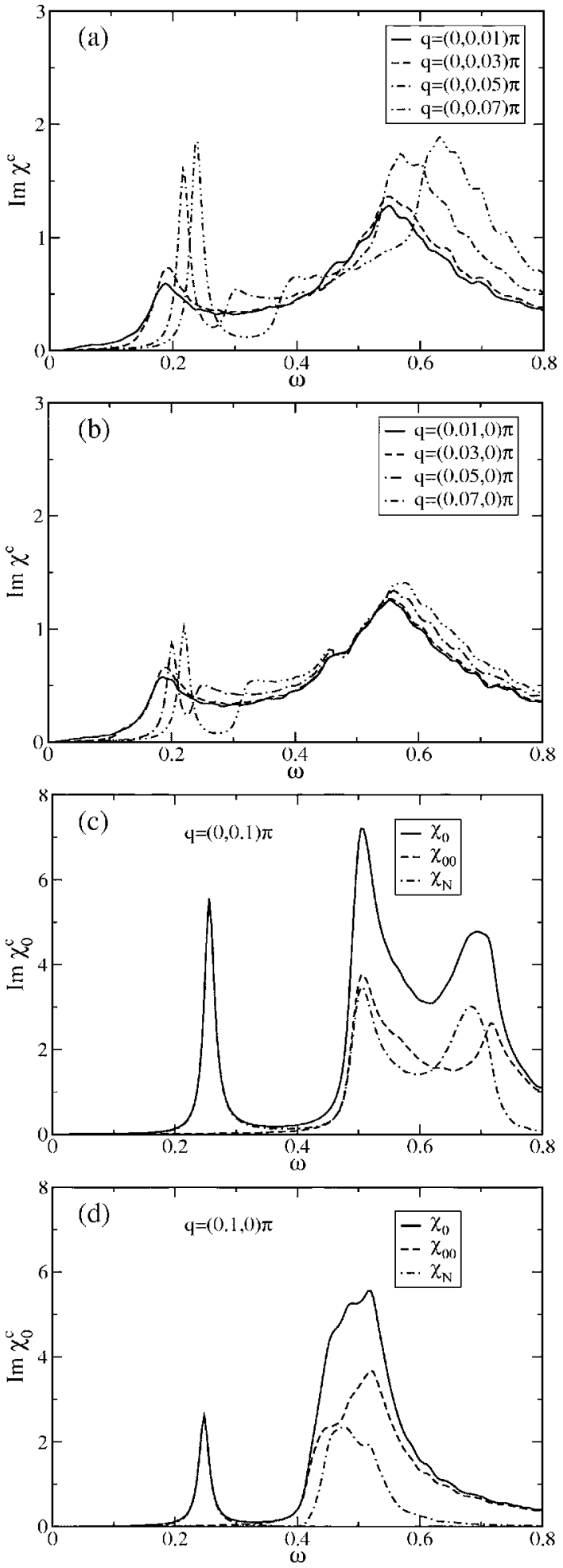

FIG. 3. Imaginary part of the charge susceptibilities. (a) and (b) are plots of the RPA susceptibilities $\operatorname{Im} \chi^{c}$ in the $q_{x}$ and $q_{y}$ directions with different $|q|$ values. (c) and (d) are the susceptibilities $\operatorname{Im} \chi_{0}^{c}$ at $\mathbf{q}=(0,0.1 \pi),(0.1 \pi, 0)$, respectively. The contribution can be separated into $\chi_{00}$, the susceptibility from the band structure, and $\chi_{N}$, the susceptibility from coupling to the nematic fluctuations. $\chi_{N}$ gives the contribution to the collective mode at lower energy. $\omega$ is in units of $t$.

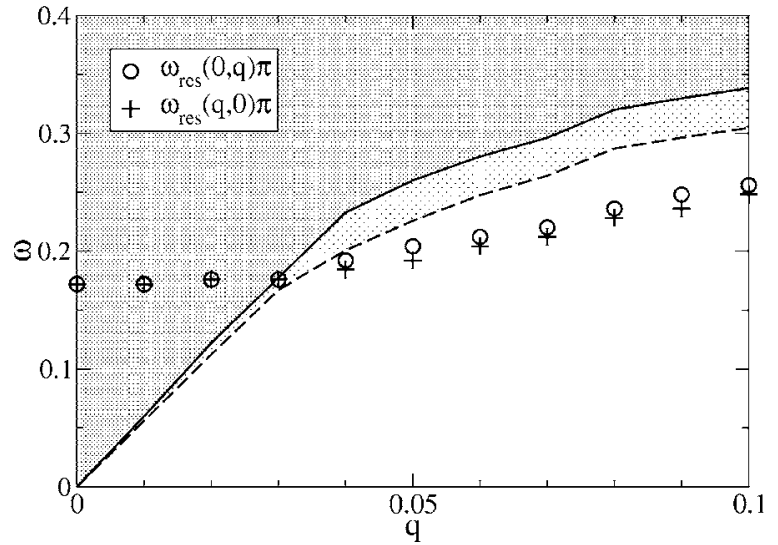

FIG. 4. The dispersion of the collective mode in $(0, q)$ (circles) and $(q, 0)$ (plus signs) directions. Shaded areas are the continuum of the excitations. The boundaries corresponds to the onset of $\operatorname{Im} \Pi_{1}^{c}$ in $(0, q)$ (solid line) and $(q, 0)$ (dashed line) directions. $\omega$ is in units of $t$.

tum dependence of $\operatorname{Im} \chi^{c}$ along the $\left(q_{x}, 0\right)$ and $\left(0, q_{y}\right)$ directions in Figs. 3(a) and 3(b). We find that the peaks become narrower with the increase of $|\mathbf{q}|$, and a sharper peak emerges at around $|\mathbf{q}| \approx 0.04 \pi$. We see that there is a clear difference for $\operatorname{Im} \chi^{c}$ in the two directions of $\left(q_{x}, 0\right)$ and $\left(0, q_{y}\right)$, which indicates broken lattice rotational symmetry due to the nematic order. To analyze the origin of the low-energy peak, we plot, in Figs. 3(c) and 3(d), $\chi_{0}, \chi_{00}$, and $\chi_{N}$ separately at $\mathbf{q}$ $=(0,0.1 \pi),(0.1 \pi, 0)$. The bare charge susceptibility $\chi_{00}$ contributes mainly to the broader spectrum at high energies with different spectral weights for $(0,0.1 \pi)$ and $(0.1 \pi, 0)$. The coupling of the density to the nematic order fluctuations provides a well-defined resonance peak at low energy, in addition to the high-energy spectrum. This corresponds to the amplitude mode of the nematic fluctuations. Comparing Figs. $3(\mathrm{c})$ and $3(\mathrm{~d})$, the anisotropy between the wave vectors, $(0,0.1 \pi)$ and $(0.1 \pi, 0)$, is manifested in the amplitudes of the resonance peak and the weight and shape of the highenergy spectrum.

To understand the dispersion relation of the amplitude mode, we summarize the dispersion of the mode in Fig. 4. The resonance frequency $\omega_{\text {res }}$ is given by the peak position of $\operatorname{Im} \chi^{c}$. The shaded areas correspond to a continuum of excitations and the boundaries are given by the onset of $\operatorname{Im} \Pi_{1}^{c}(\mathbf{q}, \omega)$. We find that the collective mode is overdamped in the small- $q$ region and becomes a well-defined mode at moderate $q$. We also see that when the mode becomes sharp, there exists a weak anisotropy in the resonance frequencies in the two different directions of momentum transfer, $|\mathbf{q}|$. This sharp mode is the collective mode associated with the nematic order, so it can provide a direct observation for the nematic order in the real materials. ${ }^{23}$

\section{DISCUSSION}

The incommensurate spin susceptibility obtained from our calculation based on the idea of coexisting nematic and superconducting orders shows great similarities to the experi- 
mental magnetic scattering pattern reported in a recent inelastic neutron experiment: ${ }^{10}$ while the basic character of spin excitations is two dimensional, the amplitude and width of the incommensurate peaks along the locus of maximum spin spectral weight is modulated in a one-dimensional fashion. We find that at constant-frequency scan, the amplitude of the spectral weight is maximum at incommensurate wave vector along the $q_{x}$ direction (or $q_{y}$, depending on the direction of the Fermi surface deformation due to the nematic order), and it continues to decrease to the wave vector along the $q_{y}\left(q_{x}\right)$ direction, as shown in Fig. 1. However, the observed incommensurate fluctuations approximately form a circle (within the numerical convolution error bars) in momentum space, while our theoretical calculation shows an incommensurate pattern of diamond shape. This diamond shape feature is common to the theoretical models incorporating the RPA method. ${ }^{20,21,24}$ The anisotropy of the spectral weight depending on the direction in momentum space originates from the anisotropic band structure of the electrons due to the formation of the nematic order. The contribution from the coupling to the nematic order fluctuations for a large momentum transfer near $(\pi, \pi)$ is negligible.

On the other hand, a collective mode at moderate energies for small momentum transfer $\mathbf{q}$ is observed in the density fluctuations. Due to the opening of the superconducting gap, the space of the particle-hole continuum at low energy is limited for nodal excitations. Therefore, the collective mode becomes sharp around $|q| \approx 0.04 \pi$, while it is overdamped at $q \approx 0$ as shown in Fig. 3. The amplitude of this mode shows a strong anisotropy depending on the direction of the momentum transfer, reflecting the $x-y$ symmetry breaking nature of the nematic order, but its dispersion depends weakly on the direction of the momentum transfer. Since this is a welldefined mode and well separated from the high-energy contributions from the band structure, it can be detected by the inelastic x-ray experiments or electron scattering. It also leads to an additional signal in the optical conductivity at the frequency of the collective mode.

Another implication of the nematic ordering is the distortion of the underlying Fermi surface due to the formation of the nematic order. Angle-resolved photoemission spectroscopy shows strong in-plane anisotropy in the electronic structure, as well as in the magnitude of the superconducting gap. ${ }^{25}$ Our results suggest that the in-plane anisotropy of spin dynamics originates from the anisotropy of the hopping integrals. While the nematic order induces the hopping integral anisotropy of $t_{y} / t_{x} \sim 1.22$ with the set of parameters we used, we cannot exclude another possible explanation of the orthorhombicity of $\mathrm{CuO}_{2}$ plane $^{26}$ and its connection to the $\mathrm{CuO}$ chain in $\mathrm{YBa}_{2} \mathrm{Cu}_{3} \mathrm{O}_{6+x} \cdot{ }^{27}$ According to the band theory ${ }^{28}$ the ratio between the hopping integrals along the $x$ and $y$ directions scales roughly as $t_{y} / t_{x} \sim(b / a)^{-4}$ which is at most 0.943 around optimal doping. We find that this ratio of $t_{y} / t_{x} \approx 0.943$ for only the nearest-neighbor hopping integral cannot produce the visible anisotropy in the spin dynamics of the $d$-wave superconductor within the RPA: $t_{y} / t_{x}=0.943$ leads to a ratio of 0.91 in the peak amplitudes between $(0.42$, $0.5)$ and $(0.5,0.43)$, while our set of parameters leads to the ratio of 2.3, which depends on the strength of $F_{2}$ and $\mu$. Also, the maximum peaks are along $q_{y}$ within the orthorhom- bicity picture, while the experimental results show that the maximum peaks are along $q_{x}$.

However, this is only a quantitative argument, and further studies, such as identification of the collective modes, are required to clarify the origin of anisotropy observed in the spin excitations. It would be also interesting to study the doping dependence of the strength of anisotropy in the spin excitations. While the structural anisotropy (the ratio between the lattice constants $b$ and $a$ ) decreases as oxygen content decreases, ${ }^{29,30}$ the tendency toward nematic ordering gets stronger in the underdoped regime. ${ }^{2}$ Therefore, the strength of anisotropy in the magnetic scattering signal will either decrease or increase, depending on the origin of anisotropic hopping integrals: it gets stronger (weaker) if its origin is due to the nematic order (structural anisotropy), as the hole doping concentration decreases. While the existing data with different doping concentrations ${ }^{10,13}$ indicate a stronger anisotropy in underdoped YBCO, a systematic study is necessary to determine the origin. Another clue may come from the study on the temperature dependence of the strength of anisotropy in the magnetic scattering pattern. Since the structural anisotropy does not depend on temperature (below $700 \mathrm{~K}),{ }^{29}$ the strength of the anisotropy due to orthorhombicity depends weakly on temperature, only via the thermal broadening and temperature dependence of the superconducting order parameter. On the other hand, the anisotropy induced by the nematic order gets weaker as the temperature increases $2,13,15$ and eventually disappears at the nematicisotropic transition temperature.

\section{SUMMARY AND CONCLUSION}

While the crystalline stripes have been observed directly in maganese oxide compounds via X-ray diffraction, ${ }^{3}$ the nematic phase, which can be viewed as strongly fluctuating stripes, requires further theoretical and experimental studies to make direct connections to real materials such as hightemperature superconductors. ${ }^{2}$ Recently, scanning tunneling microscopy (STM) experiments on slightly overdoped and nearly optimally doped $\mathrm{Bi}_{2} \mathrm{Sr}_{2} \mathrm{CaCu}_{2} \mathrm{O}_{8+\delta}$ show that there exist domain structures in the local density of states (LDOS) which are very similar to the nematic state derived from a melted stripe-ordered state. ${ }^{31,32}$ Further analysis shows strong anisotropy in the LDOS modulation. These results indicate possible coexistence of the nematic order and superconductivity.

Here we have studied spin and charge susceptibilities in a superconducting phase whose underlying electronic dispersion is anisotropic due to the nematic order and aimed to understand the effects of the nematic order on spin and charge excitations at the level of weak-coupling theory. We consider a phase where $d$-wave superconducting order $\Delta_{k}$ occurs on top of the electronic nematic phase. The underlying electronic dispersion $\widetilde{\boldsymbol{\epsilon}}_{k}$ breaks the $x-y$ symmetry, and the banana shape of the energy contour for the superconducting quasiparticle, $E_{k}=\sqrt{\widetilde{\epsilon}_{k}^{2}+\Delta_{k}^{2}}$, is tilted due to the formation of the nematic order $\Delta_{N}\left(\widetilde{\epsilon}_{k}=\epsilon_{k}+\Delta_{N}\left[\cos \left(k_{x}\right)-\cos \left(k_{y}\right)\right]\right)$. The standard RPA is used to compute the spin and charge susceptibilities, where the irreducible susceptibility includes not 
only the bare one but also the coupling to the collective modes associated with the broken $x-y$ symmetry in the nematic phase.

Motivated by a recent neutron scattering experiment on detwinned optimally doped $\mathrm{YBa}_{2} \mathrm{Cu}_{3} \mathrm{O}_{6+x},{ }^{10}$ we study the spin excitations near the resonance mode $[(\pi, \pi)]$ and find that the amplitude and width of the incommensurate peaks, which appear at a set of incommensurate wave vectors $|\mathbf{q}|$ at constant frequency below the resonance, depend on the direction of the momentum transfer. For example, a pronounced anisotropy in the amplitude of incommensurate peaks between $\left(\pi, \pi \pm q_{y}\right)$ and $\left(\pi \pm q_{x}, \pi\right)$ where $q_{x} \approx q_{y}$ has been found, which shows close similarities to the magnetic scattering pattern observed in the neutron scattering experiment. Our analysis shows that the main contribution of this anisotropy stems from the anisotropic band structure due to the nematic order. However, to clarify the origin of the anisotropic band dispersion, further studies, such as temperature and doping dependence of the anisotropy in the signal strength, are required.

In the charge channel, the coupling between the density and collective modes shows a well-defined collective mode for small momentum transfer. While this mode is overdamped due to the particle-hole continuum near $q \approx 0$, it becomes sharp as $q$ increases, due to fact that the space of Landau damping is restricted to the small momentum for low energy because of the opening of the superconducting gap. This mode can be detected by inelastic x-ray scattering and may lead to additional signal in optical conductivity.

It is interesting to note that the form factors for the nematic fluctuations are very similar to those of the Raman vertices for the $B_{1 g}$ and $B_{2 g}$ channels, and thus the collective mode in the charge susceptibility will produce an additional Raman response in the particular channels. Furthermore, we expect to find interesting features of nematic order in the LDOS modulations as observed in STM experiments. ${ }^{2}$ Also the coupling of the nematic fluctuations to the lattice degrees of freedom may result in some anomalous phonon responses. These are very interesting questions and are the subjects of future studies.

\section{ACKNOWLEDGMENTS}

We thank Steven A. Kivelson, B. Buyers, B. Keimer, and D. Manske for valuable discussions. We also thank K. Sengupta for a critical reading of the manuscript. This work was supported by NSERC of Canada, Canada Research Chair, Canadian Institute for Advanced Research, Alfred P. Sloan Foundation (H.Y.K), and Emerging Material Knowledge program funded by Materials and Manufacturing Ontario (Y.J.K, H.Y.K).
${ }^{1}$ S. A. Kivelson, E. Fradkin, and V. J. Emery, Nature (London) 393, 550 (1998).

${ }^{2}$ S. A. Kivelson, E. Fradkin, V. Oganesyan, I. P. Bindloss, J. M. Tranquada, A. Kapitulnik, and C. Howald, Rev. Mod. Phys. 75, 1201 (2003).

${ }^{3}$ S. Mori, C. H. Chen, and S. W. Cheong, Nature (London) 392, 473 (1998).

${ }^{4}$ M. P. Lilly, K. B. Cooper, J. P. Eisenstein, L. N. Pfeiffer, and K. W. West, Phys. Rev. Lett. 82, 394 (1999).

${ }^{5}$ R. R. Du, D. C. Tsui, H. L. Stormer, K. B. Cooper, L. N. Pfeiffer, K. W. Baldwin, and K. W. West, Solid State Commun. 109, 389 (1999).

${ }^{6}$ K. B. Cooper, M. P. Lilly, J. P. Eisenstein, L. N. Pfeiffer, and K. W. West, Phys. Rev. B 65, 241313(R) (2002).

${ }^{7}$ J. Tranquada, B. J. Sternlieb, J. D. Axe, Y. Nakamura, and S. Uchida, Nature (London) 375, 561 (1995).

${ }^{8}$ H. A. Mook, P. Dai, F. Dogan, and R. D. Hunt, Nature (London) 404, 729 (2000).

${ }^{9}$ Y. Ando, K. Segawa, S. Komiya, and A. N. Lavrov, Phys. Rev. Lett. 88, 137005 (2002).

${ }^{10}$ V. Hinkov, S. Pailhes, P. Bourges, Y. Sidis, A. Ivanov, A. Kulakov, C. T. Lin, D. P. Chen, C. Bernhard, and B. Keimer, Nature (London) 430, 650 (2004).

${ }^{11}$ H. A. Mook, P. Dai, S. M. Hayden, G. Aeppli, T. G. Perring, and F. A. Dogan, Nature (London) 395, 580 (1998).

${ }^{12}$ Q. Si, Y. Zha, K. Levin, and J. P. Lu, Phys. Rev. B 47, 9055 (1993); P. Benard, L. Chen, and A.-M. S. Tremblay, ibid. 47, 15217 (1993); P. B. Littlewood, J. Zaanen, G. Aeppli, and H. Monien, ibid. 48, 487 (1993).
${ }^{13}$ C. Stock, W. J. L. Buyers, R. Liang, D. Peets, Z. Tun, D. Bonn, W. N. Hardy, and R. J. Birgeneau, Phys. Rev. B 69, 014502 (2004). In this paper, the anistropy of the incoomensurate peaks was found in momentum space only along two directions.

${ }^{14}$ H.-Y. Kee, E. H. Kim, and C.-H. Chung, Phys. Rev. B 68, 245109 (2003).

${ }^{15}$ I. Khavkine, C.-H. Chung, V. Oganesyan, and H.-Y. Kee, Phys. Rev. B 70, 155110 (2004).

${ }^{16}$ C. J. Halboth and W. Metzner, Phys. Rev. Lett. 85, 5162 (2000).

${ }^{17}$ V. Oganesyan, S. A. Kivelson, and E. Fradkin, Phys. Rev. B 64, 195109 (2001).

${ }^{18}$ In principle, one should diagonalize a two-by-two matrix to obtain the collective-mode spectrum; however, the off-diagonal mixing terms are small in this model, so we only need to consider the diagonal terms.

${ }^{19}$ L. P. Kadanoff and I. I. Falko, Phys. Rev. 136, A1170 (1964).

${ }^{20}$ M. R. Norman, Phys. Rev. B 61, 14751 (2000).

${ }^{21}$ J. Brinckmann and P. A. Lee, Phys. Rev. Lett. 82, 2915 (1999).

${ }^{22}$ H. J. Schulz, Phys. Rev. Lett. 64, 1445 (1990).

${ }^{23}$ H. Yamase, Phys. Rev. Lett. 93, 266404 (2004).

${ }^{24}$ Y. J. Kao, Q. Si, and K. Levin, Phys. Rev. B 61, R11898 (2000).

${ }^{25}$ D. H. Lu et al., Phys. Rev. Lett. 86, 4370 (2001).

${ }^{26}$ I. Eremin and D. Manske, Phys. Rev. Lett. 94, 067006 (2005); the anisotropic factor was applied to all hopping integrals $\left(t_{1}, \ldots, t_{5}\right)$.

${ }^{27}$ T. Zhou and J.-X. Li, Phys. Rev. B 69, 224514 (2004).

${ }^{28}$ O. K. Andersen, A. I. Liechtenstein, O. Jepsen, and F. Paulsen, J. Phys. Chem. Solids 56, 1573 (1995).

${ }^{29}$ J. D. Jorgensen, B. W. Veal, A. P. Paulikas, L. J. Nowicki, G. W. 
Crabtree, H. Claus, and W. K. Kwok, Phys. Rev. B 41, 1863 (1990).

${ }^{30}$ H. Casalta, P. Schleger, P. Harris, B. Lebech, N. H. Andersen, R. Liang, P. Dosanjh, and W. N. Hardy, Physica C 258, 321 (1996).
${ }^{31}$ C. Howald, H. Eisaki, N. Kaneko, M. Greven, and A. Kapitulnik, Phys. Rev. B 67, 014533 (2003).

${ }^{32}$ C. Howald, H. Eisaki, N. Kaneko, and A. Kapitulnik, Proc. Natl. Acad. Sci. U.S.A. 100, 9705 (2003). 